\title{
Average reference recording from the vagal nerve reveals an evoked indirect response
}

\author{
S.C.M.A. Ordelman, L. Kornet, R. Cornelussen, H.P.J. Buschman, P.H.Veltink
}

\author{
S.C.M.A. Ordelman, P.H.Veltink \\ Institute for Biomedical Technology \\ University of Twente \\ Enschede, the Netherlands
}

\author{
L. Kornet, R. Cornelussen, H.P.J. Buschman \\ Medtronic \\ Maastricht/Heerlen, the Netherlands
}

\begin{abstract}
The vagal nerve conveys information from and to many organs. The components of the compound action potentials (CAPs) recorded from the vagal nerve give information about the different fiber types present. In this paper, we show that with the average reference recording method an additional component can be measured. This component is missed when using a tripolar recording method. Recordings were made in anesthetized pigs. Results show that there is an indirect component in the CAP of the cervical vagal nerve. This component comes from the periphery, possibly from the heart.
\end{abstract}

Keywords-vagal nerve; stimulation; nerve fibers; compound action potentials

\section{INTRODUCTION}

The vagal nerve is part of the parasympathetic nervous system and innervates many organs. The vagal nerve is a possible source of information about these organs. The response of fibers in the vagal nerve to electrical stimulation has been studied in both animals and humans [1-4]. Compound action potentials (CAPs) with components coming from thick, myelinated fibers to thin, unmyelinated fibers are identified. The thickest fibers have the highest conduction velocity, up to $70 \mathrm{~m} / \mathrm{s}$ [3]. The thinnest fibers have the lowest conduction velocity, as low as $1.2 \mathrm{~m} / \mathrm{s}[1]$.

When recording the compound action potentials, at least two electrode configurations are needed. One electrode configuration for stimulating and one electrode configuration for recording. These two electrode configurations are placed on the same nerve with some distance in-between, usually a few centimeters. This distance needs to be large enough to ensure that the stimulus artifact does not obscure the components of the compound action potentials in the recordings, and that the various components do not overlap. In previous studies [1-4] the distance between stimulation and recording site was divided by the time between the stimulus and the recorded components of the CAP to determine the conduction velocities. It was assumed that each component of the CAP was a direct effect of the stimulus applied to the nerve. Hereby, the possibility of an indirect component was excluded. In our study, the possibility is addressed of an indirect component in the CAP which travels via the brainstem or the periphery. By using the average reference recording method [5] signals can be recorded from individual electrode sites within the cuff. This enables us to determine the direction and velocity of a signal within a cuff. Also, it eliminates the need for a tripolar configuration. The disadvantage of using a tripolar configuration is that it applies a second order spatial derivative. This second order spatial derivative strongly reduces dispersed signals, as will be illustrated in the results.

\section{METHODS}

\section{A. Experimental setup}

The experiments were performed in seven female Dutch Landrace pigs. The pig was premedicated with $0.5 \mathrm{mg} / \mathrm{kg}$ Morphine IM 20 minutes before induction. Zoletil (Telazol), $3 \mathrm{mg} / \mathrm{kg}$, IM and xylazine $0.5 \mathrm{mg} / \mathrm{kg}$, IM were administered. Isoflurane was maintained once the pig was intubated, the IV line was placed and the paws were shaved for fitting ECG electrodes. The pig was anesthetized with a combination of isoflurane $(0-1.5 \%)$ and fentanyl $\left(6-10 \mu \mathrm{g} \mathrm{kg}^{-1} \mathrm{hr}^{-1}\right)$. At the end of the experimental day, the pig was euthanized with an overdose of pentobarbital.

The left and right vagal nerves were dissected free from the surrounding tissue and specially made self-coiling cuff electrodes were placed for recording and stimulation. Each electrode was $15 \mathrm{~mm}$ long and had three circular Pt/Ir electrode contacts with an interelectrode distance of $4 \mathrm{~mm}$. The cuff was made in various diameter ranges, to ensure a proper fit around the nerve.

ENG (electro neurogram) signals from the vagal nerves were amplified by a TMSi Refa amplifier (TMS international, Oldenzaal, The Netherlands) and ECG and blood pressure recordings were amplified with a TMSi Porti amplifier (TMS

This research is sponsored by Medtronic 
international, Oldenzaal, The Netherlands). A sampling frequency of $20 \mathrm{kHz}$ was used for the ENG. Signals were measured using the average reference method. The average reference method used the average of all the recorded channels as a reference [5]. It eliminated the need for a bipolar or tripolar recording configuration and allowed for the simultaneous recording of ENG signals from all three electrode sites on the cuff. Data storage was done with a laptop and PortiLab2 software (TMS international, Oldenzaal, The Netherlands).

For recording indirect components, the left vagal nerve was stimulated while the right vagal nerve was sensed and vice versa. Also, simultaneous recordings from the left and right vagal nerves were performed while stimulating the left vagal nerve.

Nerve stimulation was performed with a specially made stimulator. Stimulation with individual pulses $(1 \mathrm{~Hz})$ at various pulse widths and amplitudes was used to evoke CAPs. Stimulation parameters ranged from $35-500 \mu$ s for the pulse width, and $0.1-5 \mathrm{~mA}$ for the pulse amplitude.

\section{B. Signal analysis}

Offline signal analysis was performed in Matlab R2007a (The Mathworks Inc.). The common signal for each electrode cuff was subtracted from the individual electrode sites on that cuff. This was done to eliminate disturbing common signals. A tripolar derivation was obtained by subtracting the average signal of the two outer electrodes from the inner electrode in a cuff. Using the stimulation artifacts as reference points, CAPs from the same stimulation parameters in a stimulation train were ensemble averaged. In this way, an ensemble averaged CAP was obtained for each electrode site on the cuff and for the tripolar derivation of that cuff. The components of the CAP were identified from the individual electrode recordings. These components were then compared to the components in the tripolar derivation.

Conduction velocities were determined by taking the distance between consecutive recording sites and the time between recorded components of the CAPs at each of those sites. This allowed identification of the direction and velocity of the conducted CAPs along the nerve.

\section{RESULTS}

\section{A. The CAP}

Four components were classified from the CAP. A typical example is shown in figure 1. Thresholds were determined at a pulse width of $300 \mu \mathrm{s}$. The first two components were fast $\left(>40 \mathrm{~m} . \mathrm{s}^{-1}\right)$ and had a low stimulus amplitude threshold $(0.05-$ $0.4 \mathrm{~mA})$. The third component was the slowest $\left(<10 \mathrm{~m} \cdot \mathrm{s}^{-1}\right)$ and had a higher threshold (1.2-2.9 mA). The fourth component had a similar threshold value to the second component $(0.05$ $0.5 \mathrm{~mA}$ ), but a longer latency (figure 1). The fourth component occurred with a small latency on all consecutive recording sites, showing that it had a high velocity within the electrode cuff $\left(>40 \mathrm{~m} . \mathrm{s}^{-1}\right)$. The long time between stimulus and recording and high velocity within the cuff show that this fourth component cannot be a direct response to stimulation, but travelled a longer path before entering the cuff. Therefore, it must be an indirect component.

Since the fourth component has a small latency between consecutive electrode sites, and is dispersed, the amplitude varies little between the three electrode sites within a cuff. For this reason, when taking a second order spatial derivative, as is done with a tripolar configuration, the fourth component is strongly reduced (figure 2).

\section{B. The indirect component}

To further investigate the nature of the fourth component, the left vagal nerve was stimulated while recording from the right vagal nerve and vice versa. Then, a CAP was visible in the other vagal nerve. Since this cannot be the result of direct stimulation of the other vagal nerve, this must be an indirect component. The indirect component also occurred, with a similar latency, in the nerve that was stimulated itself (the fourth component in figure 1), which showed that it was conveyed through both the left and the right vagal nerve simultaneously. The indirect component was still present after the left vagal nerve was cut centrally, but disappeared after the nerve was subsequently cut peripherally. This showed that the indirect component does not originate from the brainstem, but came from the periphery.

\section{DISCUSSION}

Indirect responses to stimulation were found in the pig's cervical vagal nerves by using the average reference method as a novel way to look at the evoked compound action potentials. These indirect responses may have been misclassified as a slow response of C-fibers by other researchers, or missed completely due to the use of a tripolar configuration. The indirect response appeared at the different recording sites along the nerve with a short time in-between, showing that it has a high velocity. The indirect response was still observed after the nerve was centrally cut and disappeared after the nerve was peripherally cut, showing that it did not originate in the brain stem but came from the periphery. A possible explanation is that it originated from the neural network on the heart, which has been named the 'little brain on the heart' [6]. This is feasible, since afferent cardiac fibers can have a velocity comparable to the one found for the indirect component [7]. Additional research is needed to further assess the importance of this indirect component in relation to the heart.

The average reference method was used for recording the compound action potentials. When using this method, the average of all recorded channels is subtracted from each channel separately. Therefore, when recording from several electrode cuffs at the same time, signals from different channels can influence each other. This problem was overcome by recording from one electrode cuff at a time. If more than one cuff is measured simultaneously, the average of the three signals of one cuff was used in offline analysis as a reference for the signals in that cuff. In that way, disturbances coming from signals in the other cuffs are eliminated. 


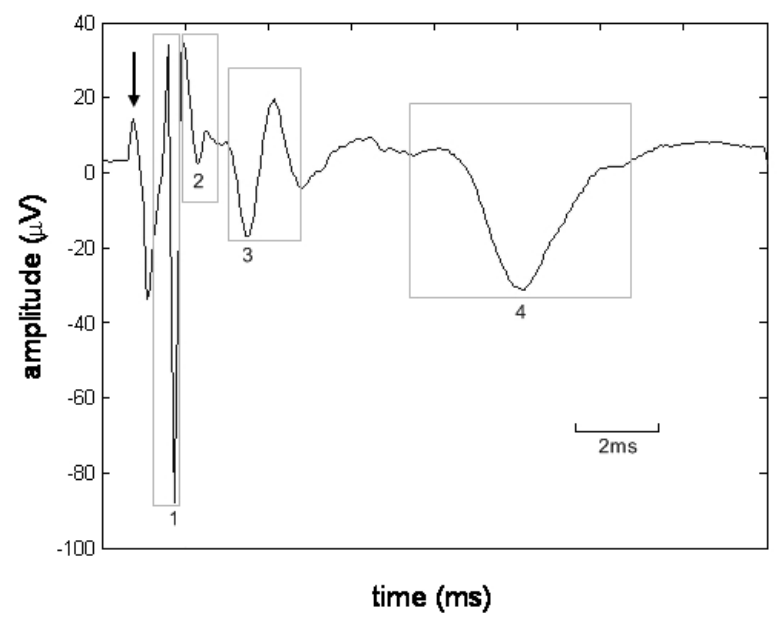

Figure 1. A typical example of an ensemble averaged compound action potential (average of $120 \mathrm{CAPs}$ ) with the four components identified. The arrow indicates the stimulus artefact.

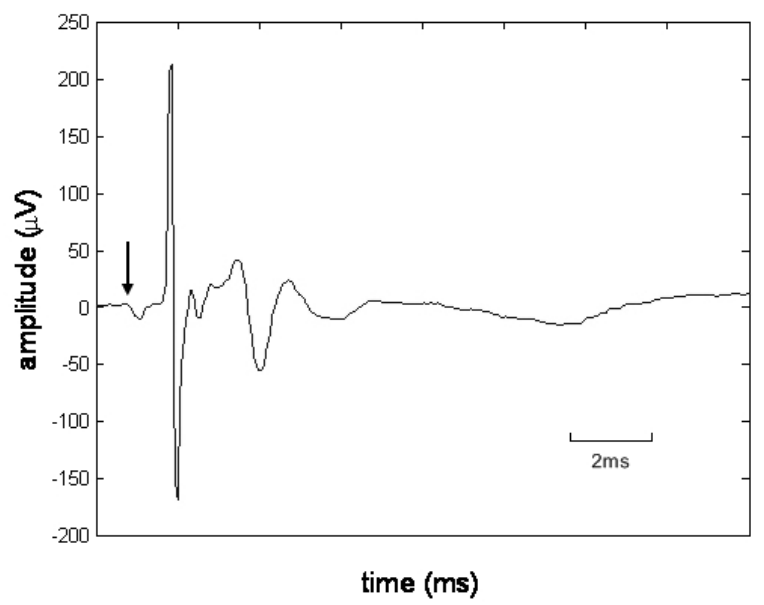

Figure 2. The same signal as shown in figure 1, but now with a tripolar configuration. The fourth component nearly completely disappears.

\section{ACKNOWLEDGMENT}

The authors gratefully acknowledge the assistance during the experiments of Joyce Suyk.

\section{REFERENCES}

[1] K.J. Deneef, J.R.C. Jansen, and A. Versprille, "Developmental Morphometry and Physiology of the Rabbit Vagus Nerve," Dev Brain Res, vol. 4,1982, pp. 265-274

[2] M.S. Evans, S. Verma-Ahuja, D.K. Naritoku, J.A. Espinosa, "Intraoperative human vagus nerve compound action potentials," Acta Neurol Scand, vol. 110, 2004, pp. 232-238

[3] M. Tosato, K. Yoshida, E. Toft, V. Nekrasas, J.J Struijk, "Closed-loop control of the heart rate by electrical stimulation of the vagus nerve," Med Biol Eng Comput, vol. 44, 2006, pp. 161-169

[4] A. Vuckovic, J.J. Struijk, and N.J.M. Rijkhoff, "Diameter selective nerve fiber stimulation in the vagal nerve using anodal block, depolarising prepulses and long exponentially rising pulses," in 9th Annual Conference of the International FES Society, 2004, Bournemouth, UK.

[5] F.F. Offner, "The Eeg as Potential Mapping - the Value of the Average Monopolar Reference," Electroen Clin Neuro, vol. 2, 1950, pp. 213-214

[6] J.A. Armour, "The little brain on the heart," Clev Clin J Med, vol. 74, 2007, pp. S48-51

[7] E. Agostoni, J.E. Chinnock, M.B. Daly, J.G. Murray, "Functional and Histological Studies of the Vagus Nerve and Its Branches to the Heart, Lungs and Abdominal Viscera in the Cat," J Physiol-London, vol. 135,1957, pp. $182-205$ 